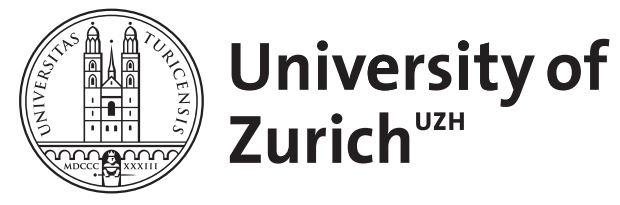

\title{
Wear and Tear Vision
}

Weber, Konrad P ; Schweier, Caterina ; Kana, Veronika ; Guggi, Thomas ; Byber, Katarzyna ; Landau, Klara

DOI: https://doi.org/10.1097/WNO.0000000000000200

Posted at the Zurich Open Repository and Archive, University of Zurich ZORA URL: https://doi.org/10.5167/uzh-107933

Journal Article

Published Version

Originally published at:

Weber, Konrad P; Schweier, Caterina; Kana, Veronika; Guggi, Thomas; Byber, Katarzyna; Landau, Klara (2015). Wear and Tear Vision. Journal of Neuro-Ophthalmology, 35(1):82-85.

DOI: https://doi.org/10.1097/WNO.0000000000000200 


\title{
Wear and Tear Vision
}

\author{
Konrad P. Weber, MD, Caterina Schweier, MD, Veronika Kana, MD, PhD, \\ Thomas Guggi, MD, Katarzyna Byber, MD, Klara Landau, MD, FEBO
}

\section{Dr. Weber:}

A 66-year-old woman was referred to the neuroophthalmology unit with a 4-month history of insidious unexplained bilateral visual loss. Ophthalmologic examination revealed visual acuity of finger counting in both eyes. Confrontation visual field testing demonstrated bilateral central scotomas with relative sparing of the peripheral fields. There was a mild right relative afferent pupillary defect. The optic discs and macule appeared normal. Specifically, there was no optic disc pallor or swelling.

The patient's medical history was significant for coronary artery disease associated with myocardial hypertrophy and for treated secondary hypothyroidism after a subtotal thyroidectomy for benign nodular disease at age 44 years. Previous surgeries included bilateral hip replacements with revision on the left side 1.5 years before consultation and a right carotid endarterectomy for a $95 \%$ stenosis 3 months before consultation. Ophthalmologic history revealed laser photocoagulation for a retinal tear in her right eye. Review of systems revealed recurrent depression, fatigue, and a decrease in appetite with weight loss of about $10 \mathrm{~kg}$. The patient also indicated that for about 3 months, she had paresthesias in her hands and feet, problems with balance, and bilateral hearing loss. She was a smoker of 10 pack-years.

At the time we examined the patient, she had already undergone brain magnetic resonance imaging (MRI), which did not reveal an explanation for her visual loss. We performed orbital MRI, with both unenhanced and contrast-enhanced sequences that showed no abnormalities either. Based on the clinical findings of profound bilateral visual loss for 4 months associated with bilateral central scotomas and a normal fundus examination, normal neuroimaging, and significant constitutional symptoms, we suspected that the patient had paraneoplastic retinopathy,

Departments of Ophthalmology (KPW, CS, KL) and Neurology (KPW, VK), University Hospital Zurich, Zurich, Switzerland; Department of Orthopedics (TG), Schulthess Clinic, Zurich, Switzerland; Division of Occupational and Environmental Medicine Division (KB), University of Zurich and University Hospital Zurich, Zurich, Switzerland.

K. P. Weber acts as an unpaid consultant and has received funding for travel from GN Otometrics. The remaining authors report no conflicts of interest.

Address correspondence to Klara Landau, MD FEBO, Department of Ophthalmology, University Hospital Zurich, Frauenklinikstrasse 24, CH-8091 Zürich, Switzerland; E-mail: klara.landau@usz.ch such as cancer-associated retinopathy (CAR) or melanomaassociated retinopathy (MAR). A full-field electroretinogram (ERG) according to ISCEVstandards revealed severe cone dysfunction (amplitude of single flash b-wave and 30$\mathrm{Hz}$ flicker response reduced to $20 \%-30 \%$ of the normal mean) and low normal rod function (amplitude of the bwave reduced to $30 \%-40 \%$ of the normal mean).

\section{Dr. Landau:}

In view of the ERG findings that seemed to be consistent with a paraneoplastic retinopathy, we referred the patient for a systemic evaluation.

\section{Dr. Weber:}

An extensive workup revealed no evidence of malignancy. In addition, an assay for antirecoverin antibodies for CAR was negative, as were assays for a variety of anti-central nervous system antibodies, including $\mathrm{Hu}, \mathrm{Ri}, \mathrm{Yo}$, Amphiphysin, CV2, Ta/Ma2, and Ma. A complete blood count was normal except for polycythemia with hemoglobin of $185 \mathrm{~g} / \mathrm{L}$ (normal: 117-153 g/L). Serum vitamin $\mathrm{B}_{12}$ concentration was normal. The erythrocyte sedimentation rate was $2 \mathrm{~mm} / \mathrm{h}$, and autoimmune screening including ANA, ANCA, anti-SS-A, and anti-SS-B revealed no abnormalities. Both gynecologic and dermatologic examinations were nonrevealing. A FDG-PET-CT showed inflammation of the left hip consistent with bursitis. We elected to follow the patient; however, she was lost to follow-up.

\section{T. Guggi:}

Two years later, the patient was examined in our orthopedic clinic for chronic left hip pain and muscle tenderness that had persisted after the replacement of a fractured ceramic head 3 years earlier. Radiography of the left hip revealed what appeared to be metallic debris around the prosthesis (Fig. 1). Consequently, the patient underwent revision of the prosthesis. After removal of the cup and the deteriorated metal head, the site was thoroughly debrided. A new cup with a cross-linked polyethylene insert was implanted, and a ceramic head with metal head adapter replaced the removed metal head. The original stem was left in place. On inspection at the time of surgery, the retrieved metal head was worn down (Fig. 2), and there was a significant 

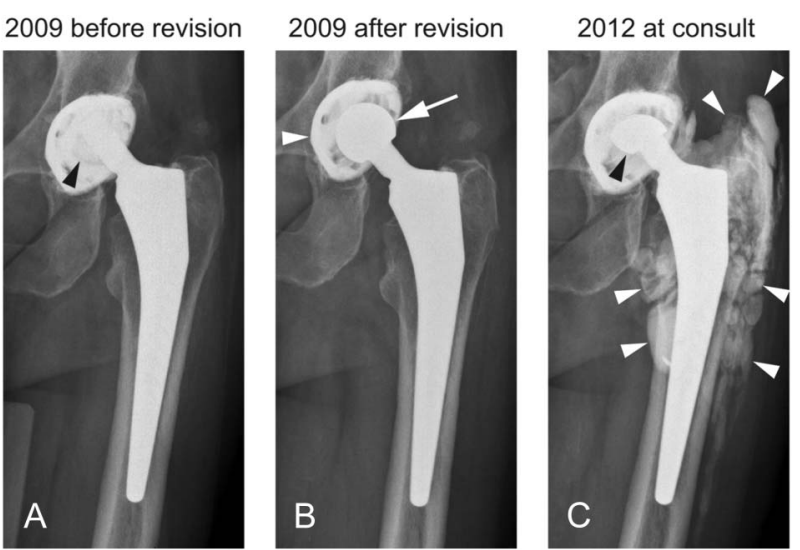

FIG. 1. A. Radiology of the left hip shows a fractured ceramic head (arrowhead) prompting revision of the prosthesis. B. During revision, the ceramic head was replaced with a CoCrMo metal head (arrow) and a new polyethylene liner (arrowhead). C. Radiography obtained for chronic hip pain 3 years later reveals a worn-down metal head (black arrowhead) with radio-opaque debris (white arrowheads).

amount of metallic debris surrounding the prosthesis (Fig. 3). A blood test was performed.

\section{Dr. Byber:}

The blood test was an assay for serum cobalt. This revealed an extremely elevated serum cobalt level of $6975 \mathrm{nmol} / \mathrm{L}$ (normal: $<17 \mathrm{nmol} / \mathrm{L}$ ), so that a diagnosis of cobalt neurotoxicity was made. After surgery, cobalt levels exponentially dropped to $1220 \mathrm{nmol} / \mathrm{L}$ within 40 days and to 136 $\mathrm{nmol} / \mathrm{L}$ within a year, approaching the reference value of $119 \mathrm{nmol} / \mathrm{L}$ for patients with metal implants. This decrease corresponded well to the known toxicokinetics of the metal

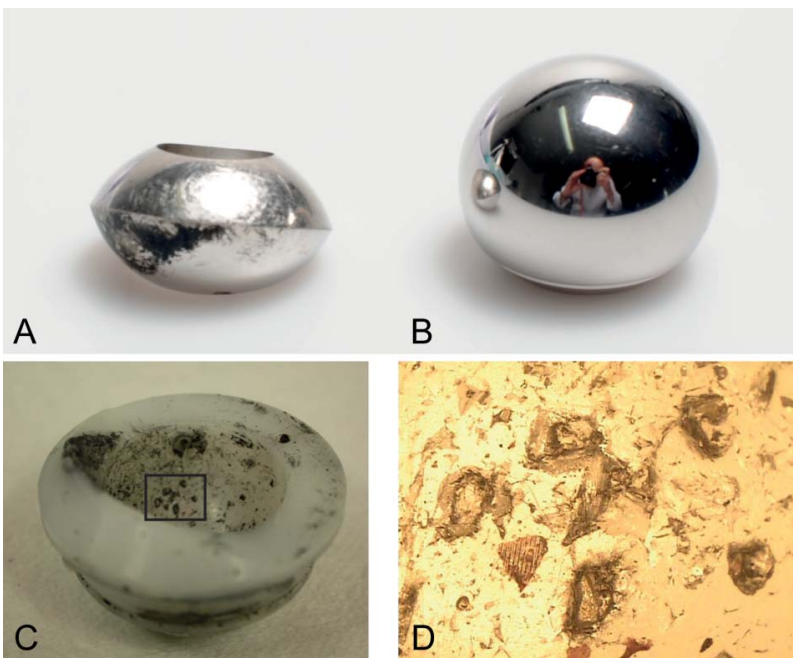

FIG. 2. A. Worn-down CoCrMo metal head of the implanted hip prosthesis compared with a new metal head $(\mathbf{B})$, of the same type. C. Worn-down polyethylene inlay with ceramic particles imprinted in the cavity. D. Imprinted ceramic splinters on the polyethylene inlay in the area indicated in (C), creating an abrasive surface.

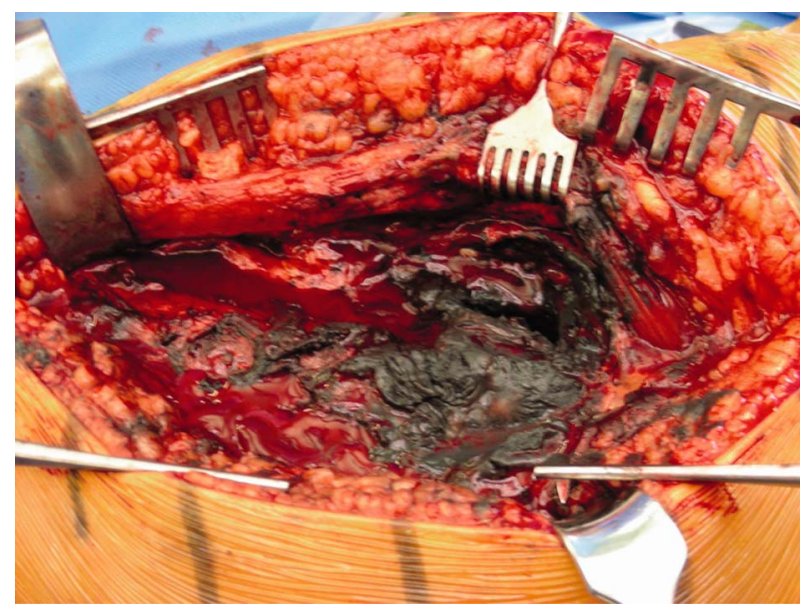

FIG. 3. Surgical site during revision of the left hip reveals significant metallic debris around the prosthesis.

(1), consistent with the hypothesis that the defective prosthesis was the cause for the elevated cobalt level.

\section{Final Diagnosis}

Cobalt neurotoxicity from abraded metallic hip prosthesis.

\section{Dr. Weber:}

After removal of the prosthesis, the patient's hip pain slowly improved such that she was able to walk without discomfort. Her hearing, which had been slowly worsening while the damaged prosthesis was in place, also improved (Fig. 4). Unfortunately, her visual acuity did not improve remaining finger counting in each eye.

\section{Dr. Weber and Dr. Landau:}

In our patient's case, cobalt neurotoxicity caused devastating visual loss accompanied by a dramatic deterioration of the patient's general condition triggered by a mechanical orthopedic problem. Over the last few years, reports of cobalt toxicity related to hip implants have attracted increasing medical attention $(2,3)$ as exemplified in the television program "House" (4). In terms of visual loss, case reports suggest that cobalt poisoning may induce toxic effects in both retinal ganglion cells and optic nerve axons $(5,6)$. In our patient, the retinopathy was the cardinal symptom, whereas the optic neuropathy only became apparent on follow-up. The toxicity seems to be caused primarily through interaction of free $\mathrm{Co}$ (II) ions with various metabolic processes (1). Interestingly, cobalt chloride has been used as an hypoxia-mimicking agent in a murine model of retinal photoreceptor cell degeneration (7).

Cobalt alloys have been widely used as implant materials for more than 80 years due to their high specific strength. Rarely, patients with metal-containing prostheses develop metallosis, a local reaction of soft tissue surrounding the implant that is characterized by the formation of a black- 


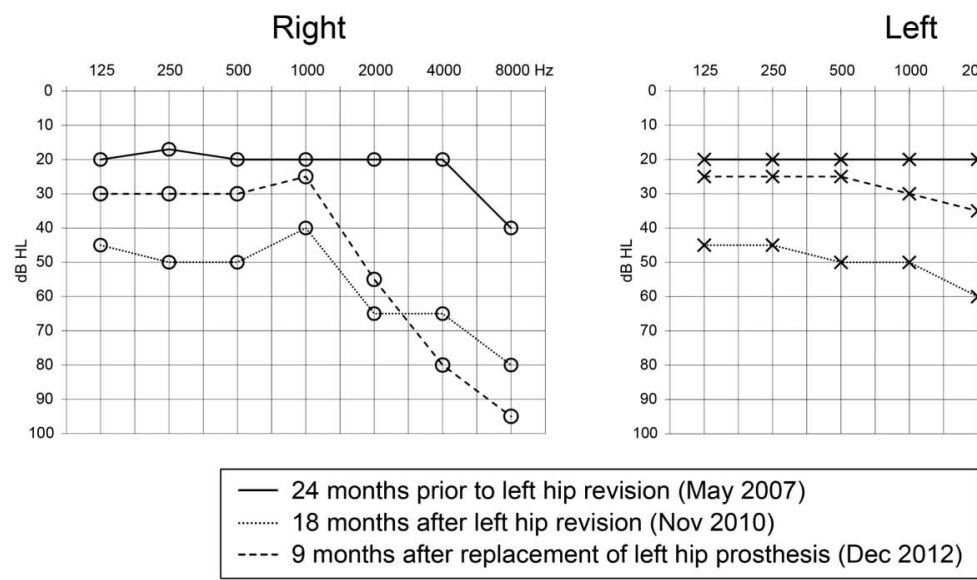

FIG. 4. Sequential audiograms show severe bilateral hearing loss with some recovery after replacement of hip prosthesis. Hearing loss 24 months before left hip revision: right $=7 \%$, left $=9 \%$; hearing loss 18 months after left hip revision: right $=$ $61 \%$ left $=66 \%$; hearing loss 9 months after replacement of left hip prosthesis right $=47 \%$, left $=28 \%$ (results according to Current Procedural Terminology of the American Medical Association).

stained "pseudotumor" (8). In our case, the circumstances leading to the toxic metallosis in the hip were explained by the sequence of implants used (9) (Fig. 1). Initially, the hip prosthesis was revised because of a fracture of the ceramic femoral head (10). The ceramic head was replaced with a metal head paired with a polyethylene inlay. Subsequently, fine ceramic particles left behind from the broken head adhered to the polyethylene inlay and grinded down the new metal head like a grindstone (Fig. 2 and Supplemental Digital Content, Fig. E1, http://links.lww.com/WNO/A132). The combination of incompatible implants violated a major rule for orthopedic surgeons: never mix but always match orthopedic components (11).

In hindsight, the deterioration of the patient's general condition, including many of her varied symptoms and signs, were attributable to cobalt toxicity (1). The patient not only experienced vision loss but also had both hearing impairment and imbalance, both of which improved after replacement of the prosthesis (Figs. 4 and 5). Although the patient was never formally tested, her acral paresthesias may have been due to a cobalt-induced polyneuropathy.

Two helpful laboratory signs of cobalt toxicity are polycythemia and hypothyroidism, both of which were present in our patient (although the hypothyroidism may have been related solely to her previous thyroidectomy). Polycythemia seems to be a particularly sensitive laboratory parameter for this condition (8), as it is rarely present otherwise in elderly patients with hip implants. Therefore, it may be helpful to test for polycythemia in patients with cobaltcontaining implants.

Neurological complications from elevated cobalt levels in patients with orthopedic implants are rare. In previous reports $(5,6)$, cobalt concentrations varied between 2074 $\mathrm{nmol} / \mathrm{L}$ (serum measurements) and 10,625 $\mathrm{nmol} / \mathrm{L}$ (whole blood measurements). Cobalt concentrations can be measured in serum or in whole blood; however, in patients with long-term exposure, the serum and blood cobalt concentrations will be dissimilar, as the metal ions are incorporated into the red blood cells (8). Therefore, whole blood measurements should be performed in such cases, as they are more representative of the actual cobalt level.

Our patient had a hypertrophic cardiomyopathy associated with congestive heart failure and a pericardial effusion. Two years after the initial left hip revision, she had undergone a muscle biopsy from the right ventricle that showed cardiomyocyte degeneration and atrophy as well as

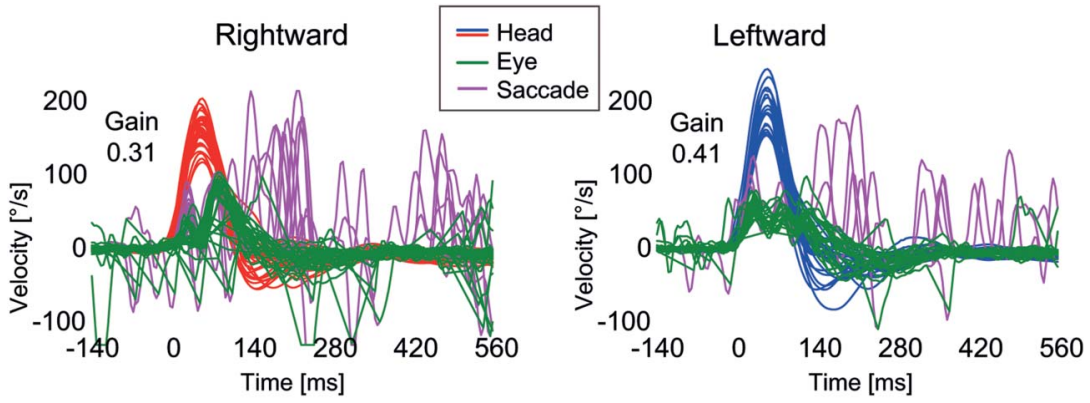

FIG. 5. Video head impulse test obtained 1.5 years after replacement of left hip prosthesis demonstrates bilaterally reduced vestibulo-ocular reflex gains (normal gain $>0.8$ ) with compensatory catch-up saccades (purple spikes) in response to head rotations to either side indicating involvement of the vestibular system. 

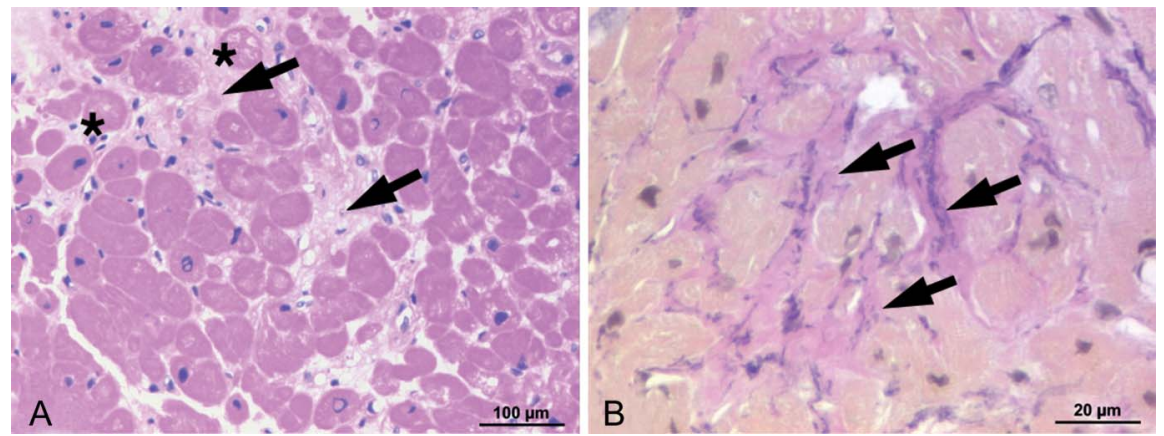

FIG. 6. Myocardial biopsy performed 2 years after left hip revision. A. There is myocyte degeneration and atrophy (arrows) and necrotic myocytes with vacuolation of the cytoplasm (asterisks) consistent with cardiomyopathy (hematoxylin \& eosin). B. Myocardial fibrosis (arrows) is also present (elastica van Gieson). Additional stainings showed no signs of storage disease or amyloidosis (not shown). The findings are not specific but consistent with cobalt cardiotoxicity.

mild fibrosis (Fig. 6). As these findings were nonspecific (12), the suspicion of cobalt cardiac toxicity was not raised at that time; however, in retrospect, it, too, was caused by cobalt toxicity. The peculiar cobalt-induced cardiomyopathy observed in our patient first came to medical attention as "Quebec beer drinker's cardiomyopathy" (13). In 1965, the addition of cobalt sulfate as a foam stabilizer in beer triggered an epidemic of 48 cases of cardiomyopathy associated with acute congestive heart failure within 9 months. The epidemic only affected heavy beer drinkers (3-6 L/d) of a particular brand and individuals with poor nutritional status. Interestingly, in this acute setting with a mortality of $40 \%$, visual loss did not occur or perhaps simply was overlooked under these dramatic circumstances (14).

Recovery of vision has been reported in cobalt ocular toxicity after a decrease in ion concentration (15). Although cobalt levels dropped rapidly during the months after hip revision in our patient, her vision did not improve and optic atrophy developed. The patient's course thus suggests that, as in other cases of toxic retinopathy or optic neuropathy, the effects may be irreversible when toxicity is severe and longstanding. However, improvement of her hearing, along with the recovery of her general health, is consistent with cobalt intoxication.

\section{CONCLUSIONS}

Through a chain of unfortunate circumstances, a mechanical orthopedic problem turned into a devastating visual loss in this case. Given the high prevalence of hip implants, every neuro-ophthalmologist should keep cobalt neurotoxicity in mind and ask patients with obscure visual loss about hip replacements. If cobalt intoxication is suspected, measurements of cobalt concentration should be prompted.

\section{ACKNOWLEDGMENTS}

The authors thank the patient for her cooperation and acknowledge the help of all the many physicians involved in treating the patient. The authors thank Dr Kathleen Digre for her helpful comments and Patrick Hofmann for providing part of the image material.

\section{REFERENCES}

1. Paustenbach DJ, Tvermoes BE, Unice KM, Finley BL, Kerger BD. A review of the health hazards posed by cobalt. Crit Rev Toxicol. 2013;43:316-362.

2. Rizzetti MC, Liberini P, Zarattini G, Catalani S, Pazzaglia U, Apostoli P, Padovani A. Loss of sight and sound. Could it be the hip? Lancet. 2009;373:1052.

3. Allen LA, Ambardekar AV, Devaraj KM, Maleszewski JJ, Wolfel EE. Clinical problem-solving. Missing elements of the history. N Engl J Med. 2014;370:559-566.

4. Dahms K, Sharkova Y, Heitland P, Pankuweit S, Schaefer JR. Cobalt intoxication diagnosed with the help of Dr House. Lancet. 2014;383:574.

5. Apel W, Stark D, Stark A, O'Hagan S, Ling J. Cobalt-chromium toxic retinopathy case study. Doc Ophthalmol. 2013;126:69-78.

6. Bhardwaj N, Perez J, Peden M. Optic neuropathy from cobalt toxicity in a patient who ingested cattle magnets. Neuroophthalmology. 2011;35:24-26.

7. Hara A, Niwa M, Aoki H, Kumada M, Kunisada T, Oyama T, Yamamoto T, Kozawa O, Mori $\mathrm{H}$. A new model of retinal photoreceptor cell degeneration induced by a chemical hypoxia-mimicking agent, cobalt chloride. Brain Res. 2006;1109:192-200.

8. Paustenbach DJ, Galbraith DA, Finley BL. Interpreting cobalt blood concentrations in hip implant patients. Clin Toxicol. 2014;52:98-112.

9. Rizzetti MC, Zarattini G, Pazzaglia U, Padovani A. Blind and deaf after total hip replacement? Author reply. Lancet. 2009;373:1944-1945.

10. Allain J, Roudot-Thoraval F, Delecrin J, Anract P, Migaud H, Goutallier D. Revision total hip arthroplasty performed after fracture of a ceramic femoral head. A multicenter survivorship study. J Bone Joint Surg. 2003;85:825-830.

11. Willmann G. Ceramic cups for hip endoprotheses. 4: never mix and match [in German]. Biomed Tech (Berl). 1998;43:184-186.

12. Zywiel MG, Brandt JM, Overgaard CB, Cheung AC, Turgeon TR, Syed KA. Fatal cardiomyopathy after revision total hip replacement for fracture of a ceramic liner. Bone Joint J. 2013;95:31-37.

13. Morin Y, Daniel P. Quebec "beer-drinkers" cardiomyopathy: etiological considerations. Canad Med Assoc J. 1967;97:926-928.

14. Mercier G, Patry G. Quebec "beer-drinkers" cardiomyopathy: clinical signs and symptoms. Canad Med Assoc J. 1967;97:884-888.

15. Apel W, Stark D, O'Hagan S. An update on cobalt-chromium toxic retinopathy. Doc Ophthalmol. 2013;127:173-175. 